\title{
IS RATING OF PERCEIVED EXERTION A VALID METHOD TO MONITOR INTENSITY DURING BLOOD FLOW RESTRICTION EXERCISE?
}

original paper

( ) University School of Physical Education in Wroclaw

DOI: https://doi.org/10.5114/hm.2021.100015

\begin{abstract}
RODRIGO RAMALHO ANICETO ${ }^{1,2}$, ROBERT J. ROBERTSON ${ }^{3}$, ALEXANDRE SÉRGIO SILVA $^{2}$, PABLO B. COSTA ${ }^{4}$, LEANDRO CÂNDIDO DE ARAÚJO ${ }^{1,5}$, JULIO CESAR GOMES DA SILVA ${ }^{1,2}$, MARIA DO SOCORRO CIRILO-SOUSA ${ }^{2}$

${ }^{1}$ Study and Research Group in Biomechanics and Psychophysiology of Exercise, Department of Physical Education and Sport, Federal Institute of Education, Science and Technology of Rio Grande do Norte, Currais Novos, Brazil

2 Associate Graduate Program in Physical Education UPE/UFPB, Department of Physical Education, Federal University of Paraíba, João Pessoa, Brazil

${ }^{3}$ Center for Exercise and Health-Fitness Research, Department of Health and Physical Activity, University of Pittsburgh, Pittsburgh, USA

${ }^{4}$ Exercise Physiology Laboratory, Department of Kinesiology, California State University, Fullerton, USA

${ }^{5}$ Laboratory of Kinesiology and Biomechanics, Department of Physical Education, Integrated Colleges of Patos, Patos, Brazil
\end{abstract}

\begin{abstract}
Purpose. To examine the concurrent validity of rating of perceived exertion (RPE) in resistance exercise with blood flow restriction $(\mathrm{RE}+\mathrm{BFR})$.

Methods. Twelve trained men participated in an orientation session and 2 experimental sessions: RE + BFR and traditional resistance exercise (TRE). Arm curl and leg extension exercises were standardized by the total volume of 3 sets of 16 repetitions at 35\% of one-repetition maximum (1RM) for RE + BFR and 3 sets of 8 repetitions at 70\% of 1RM for TRE. BFR was applied to proximal positions of both the upper and lower limbs by using an elastic knee wrap with a width of $7.6 \mathrm{~cm}$. Blood lactate concentration ([La]), RPE in the active muscles (RPE-AM) and in the overall body (RPE-O) were measured at rest and the end of each set with the OMNI-RES.
\end{abstract}

Results. In RE + BFR, positive linear regression coefficients $(p<0.01)$ were found between [La] and RPE-AM (arm curl: $r=0.54$; leg extension: $r=0.71$ ) and between [La] and RPE-O (arm curl: $r=0.55$; leg extension: $r=0.74$ ). Similarly in TRE, positive coefficients $(p<0.01)$ were observed between [La] and RPE-AM (arm curl: $r=0.63$; leg extension: $r=0.63$ ) and between [La] and RPE-O (arm curl: $r=0.60$; leg extension: $r=0.59$ ).

Conclusions. The RPE scale was shown to be a valid method to monitor and regulate intensity during RE + BFR in the upper and lower limbs.

Key words: resistance training, energy metabolism, cell hypoxia, sensation

\section{Introduction}

Blood flow restriction (BFR) is a technique that, when combined with resistance exercise (RE + BFR), results in high metabolic stress. This type of exercise promotes hypoxia owing to decreased blood flow for the exercised muscles and, consecutively, increases the recruitment of type II (glycolytic) fibres, causes lactate accumulation, and prolongs metabolic acidosis via trapping and the accumulation of intramuscular protons $\left(\mathrm{H}^{+}\right.$ions, decrease in $\left.\mathrm{pH}\right)$, which stimulates metabolic receptors, possibly triggering an exaggerated acute response of the hormonal system [1,2].

Metabolite accumulation along with muscle swelling and the discomfort caused by the cuff/elastic wrap applied to the limbs in RE + BFR tend to increase

Correspondence address: Rodrigo Ramalho Aniceto, Study and Research Group in Biomechanics and Psychophysiology of Exercise, Department of Physical Education and Sport, Federal Institute of Education, Science and Technology of Rio Grande do Norte, Rua Manoel Lopes Filho, n 773, Valfredo Galvão, CEP: 59380-000, Currais Novos-RN, Brazil, e-mail: rodrigo-afa@hotmail.com

Received: July 10, 2019

Accepted for publication: April 1, 2020

Citation: Aniceto RR, Robertson RJ, Silva AS, Costa PB, De Araújo LC, Da Silva JCG, Do Socorro Cirilo-Sousa M. Is rating of perceived exertion a valid method to monitor intensity during blood flow restriction exercise?. Hum Mov. 2021;22(2):68-77; doi: https://doi.org/10.5114/hm.2021.100015. 
effort intensity. Hollander et al. [3] observed that the execution of RE + BFR by using 3 sets at $30 \%$ of onerepetition maximum (1RM) changed the rating of perceived exertion (RPE) and pain responses in a similar manner as traditional resistance exercise (TRE) performed by using 3 sets at $70 \%$ of $1 \mathrm{RM}$ during the execution of arm curl and leg extension exercises to exhaustion. Moreover, the findings of Loenneke et al. $[4,5]$ indicated that RPE was greater in $\mathrm{RE}+\mathrm{BFR}$ than in RE without BFR with the same resistance load. By contrast, other researchers believe that RE + BFR is of low intensity [1, 6-9] because of the low load applied. In this perspective, it appears that monitoring intensity during RE + BFR needs to be better elucidated.

According to the position stand of the American College of Sports Medicine [10] and various studies [11-14], RPE can be used to control and prescribe intensity in RE. RPE is a valid method to assess exercise intensity and is widely employed in RE without BFR because it is correlated with physiological variables, such as blood lactate concentration ([La]) [11, 12] and muscle activity [15]; performance variables, such as load intensity (percentage of 1RM) [16], the volume of weight lifted [12], and the prediction of 1RM [17]; and perceptual variables, such as perceived muscle pain [18]. In addition, RPE is easy to apply, inexpensive, non-invasive, and can be used to manipulate the acute variables of training without the need to conduct maximal or submaximal strength tests $[11,17]$. However, whether similar correlations are observed during $\mathrm{RE}+\mathrm{BFR}$ is unknown.

Considering that the RE + BFR training paradigm causes considerable metabolic stress and discomfort, it is possible that RPE is influenced, and, consequently, individuals may underestimate or overestimate the perceptual responses in the active muscles (RPE-AM) or in the overall body (RPE-O). In this sense, it is unclear whether RPE during RE + BFR is a valid method to monitor intensity, and this should be investigated. Notably, several studies have examined the effects of $\mathrm{RE}+\mathrm{BFR}$ on exercise intensity markers, including physiological variables (e.g., [La] and muscle activity) and perceptual responses (e.g., RPE, perceived pain and discomfort) [5, 8, 9, 19-23]. However, to our knowledge, no previous studies have investigated concurrent validation in the RE + BFR by establishing a correlation between RPE and [La]. Therefore, the aim of this study was to examine the concurrent validity of RPE for RE + BFR in the upper and lower limbs.

\section{Material and methods}

\section{Participants}

Twelve apparently healthy trained men (mean \pm standard deviation - age: $23 \pm 4$ years; body mass: $75.51 \pm 8.23 \mathrm{~kg}$; height: $1.75 \pm 0.07 \mathrm{~m}$; body mass index: $24.41 \pm 2.61 \mathrm{~kg} / \mathrm{m}^{2}$; body fat: $11.44 \pm 4.69 \%$ ) volunteered to take part in the investigation and completed all experimental procedures. The subjects were classified as trained on the basis of the regular practice of RE for at least 6 months with a minimum frequency of 3 times a week. All of the participants answered 'no' to all the questions on the Physical Activity Readiness Questionnaire (PAR-Q) and reported not having used anabolic steroids. The exclusion criteria were the use of alcohol, nicotine, drugs, or dietary supplements; musculoskeletal, cardiovascular, and cognitive complications; and the performance of any physical exercise 48 hours before the experimental sessions.

The sample size was calculated with $G^{*}$ Power version 3.1.9.2 (Franz Faul, Germany). Data from previous studies were used to calculate the statistical power necessary to establish the correlation between perceptual responses (RPE-AM and RPE-O) and the physiological marker of metabolic stress ([La]) during RE + BFR $[11,12]$. Therefore, the use of an effect size (slope H1) of 0.7 , a power of $0.80(\beta=0.20)$, and a 2-tailed significance level $(\alpha)$ of 0.05 indicated that a minimum of 11 participants were required to establish the relation between the variables from the bivariate linear regression. The calculations followed the recommendations by Beck [24] and Faul et al. [25].

\section{Experimental design}

A randomized, counterbalanced, crossover study was used to establish the concurrent validation. The participants underwent 3 sessions: an orientation session and 2 experimental sessions. In the orientation session, they underwent anthropometric measurements, a 1RM test, and familiarization with the exercises and repetition rate using a metronome, as well as received scaling instructions and exercise-memory anchoring procedures for the OMNI-RES. Next, the subjects were randomly counterbalanced to the experimental sessions: RE + BFR and TRE. In addition, they were randomly counterbalanced to the arm curl and leg extension exercises during RE + BFR and TRE. The conditions were standardized by total work (number of sets $\times$ number of repetitions $\times$ load) $[11,26]$. 
The orientation and experimental sessions occurred at the same time of day with an interval of 3-5 days between sessions. The environmental conditions were controlled and temperature was kept between 22 and $24^{\circ} \mathrm{C}$ and relative humidity between 40 and $60 \%$ [26]. Prior to each session, the participants were previously instructed to hydrate, abstain from caffeine for a minimum of 24 hours, keep their usual hours of sleep and daily activities, and report any factors/events that could affect their physical or cognitive performance (e.g., injuries and emotional problems).

\section{Orientation session}

Prior to the orientation session, the participants were instructed to abstain from exercise for a minimum of 24 hours and to eat a light meal 2 hours before the session. In this session, anthropometric measurements including height $(\mathrm{m})$, body mass $(\mathrm{kg})$, and skinfold thickness $(\mathrm{mm})$ were determined. Body mass index was calculated by dividing weight by height squared $\left(\mathrm{kg} / \mathrm{m}^{2}\right)$, and body fat percentage (\%) was estimated by using the 3-skinfold protocol by Jackson and Pollock [27]. Subsequently, the proper positioning, execution, and range of motion for the arm and leg RE were demonstrated and standardized to determine the load in the 1RM test and to familiarize the participants for future experimental sessions.

\section{One-repetition maximum test}

The load percentage used in the experimental sessions was determined by performing the 1RM test in accordance with the recommendations by Kraemer et al. [28]. The order of the tests in the exercises was the same as that used in the experimental sessions. During the 1RM test, the participants did not have visual access to exercise loads to avoid any bias in relation to the final loads applied.

\section{Familiarization}

After the 1RM test, the subjects held a session to familiarize themselves with the exercise protocol and the repetition rate of the experimental sessions. This session consisted of 2 exercise sets until concentric failure at an execution speed of 1 second for each concentric and eccentric phase and was monitored with a metronome (Korg MA30, Tokyo, Japan) [11, 16, 26], with a 5-minute rest interval between sets for both arm curl and leg extension.

\section{Rating of perceived exertion}

RPE-AM and RPE-O were measured by using the previously validated OMNI-RES [12]. The participants underwent the scaling instructions and exercise-memory anchoring procedures for the OMNI-RES during the 1RM test in the orientation session in order to obtain accuracy of the responses in the experimental sessions [29, 30]. The scaling instructions and memory-anchoring procedures were reviewed in the experimental sessions before starting the exercises, as previously described by Gearhart et al. [29] and Lagally and Costigan [30], to ensure that the participants recalled the feelings experienced in the exercise anchoring procedures performed in the orientation session.

\section{Scaling instructions}

The instructions on the scale included an explanation of the nature and use of OMNI-RES, differentiated ratings, and low and high numerical categories as scale-anchoring points. Perceived exertion was defined as the subjective intensity of effort, strain, discomfort, and/or fatigue felt during exercise [12, 29]. The OMNI-RES has 10 numerical categories (0-10), verbal and pictorial descriptors, and a scalar representation of various levels of physical exertion. A rating of 0 indicates no effort (e.g., rest), and 10 indicates maximum effort, which corresponds to a level of perception that was higher than that reported by the participants during RE at the point of muscular fatigue. The participants were instructed to rate their perception of exertion as accurately and honestly as possible and were informed that there was no right or wrong numerical category response.

\section{Anchoring procedures}

In the orientation session, exercise-memory anchoring procedures were used such that the participants could cognitively establish the perceptual ends of the OMNI-RES: rating of 1 (lower end) and rating of 9 (higher end). In the high and low anchoring procedure, the subjects were familiarized with the perceptions corresponding to a rating of 1 and 9 by performing a repetition with the minimum load available for each exercise and classifying it as 1 (extremely easy) and 9 (extremely hard) when the maximum load was obtained in the 1RM test for both RPE-AM and RPE-O. At the end of the anchoring protocol, the participants were instructed to rate their effort in the experimental sessions on the basis of the feelings experienced with responses ranging from 1 to 9 . 


\section{Blood lactate concentration}

After local antisepsis with alcohol, a 25- $\mu$ l blood sample was collected from the earlobe with heparinized capillary tubes and immediately transferred to sterile Eppendorf tubes containing $50 \mu \mathrm{l}$ of $1 \%$ sodium fluoride. All of the samples were expressed at $\mathrm{mmol} \cdot \mathrm{l}^{-1}$ by using a lactate analyser (YSI 1500 Sport Lactate Analyzer, Yellow Springs, USA) to obtain [La].

\section{Determination of blood flow restriction}

Prior to the current study, a cross-sectional study was conducted in our laboratory to determine BFR because of the limitations of previous studies. For BFR, an elastic knee wrap with a width of $7.6 \mathrm{~cm}$ (Harbinger Red-Line, Fairfield, USA) was applied to the proximal end of the upper and lower limbs (Figure 1).

For this study, the elastic wrap was fitted with a 5-cm Velcro strip at the ends to enable better fixation to the limbs (Figure 1). Assuming that the circumference of the upper and lower limbs was the greatest predictor of BFR pressure [31-33], a study was conducted among 30 participants to identify the circumference percentage that corresponded to a pressure of 7 (moderate pressure without pain) on the scale proposed by Wilson et al. [34]. First, circumference measurements in the proximal upper and lower limbs were performed. Next, the participants were provided instructions on the scale, including an explanation of the nature and use of the scale and of the low and high numerical categories as scale-anchoring points.

To low anchor, an elastic knee wrap was used with the same circumference of the arm or leg segment to familiarize the subjects with the perception equivalent to a rating of 0 (no pressure). After 1 minute, the high scale anchor was established. A fully stretched elastic knee wrap was applied to the arm or leg segment to familiarize the subjects with the perception corresponding to a rating of 10 (intense pressure with pain). Considering the circumference as a reference (100\%), the subjects were randomly assigned blind to 5 BFR conditions $(15,20,25,30$, and 35\%) and indicated on the scale a number that best corresponded to the perceived pressure. For example, a subject with an arm circumference of $30 \mathrm{~cm}$ at $20 \%$ of BFR had the elastic wrap marked with adhesive tape at $24 \mathrm{~cm}$, and the wrap was applied to the arm with this restriction of $6 \mathrm{~cm}$.

Our data demonstrated that most participants responded with a 7 (moderate pressure without pain) on the scale when their elastic wrap was set with a restriction of $25 \%$ of the circumference for the upper limbs and $30 \%$ for the lower limbs. Therefore, BFR with the elastic knee wrap was determined on the basis of the limb circumference, such that $25 \%$ was prescribed to arm curl and $30 \%$ to leg extension. The same protocol was used in the study sample during the orientation session. Moreover, in the RE + BFR session at rest, after placing the wrap with the proper restriction,
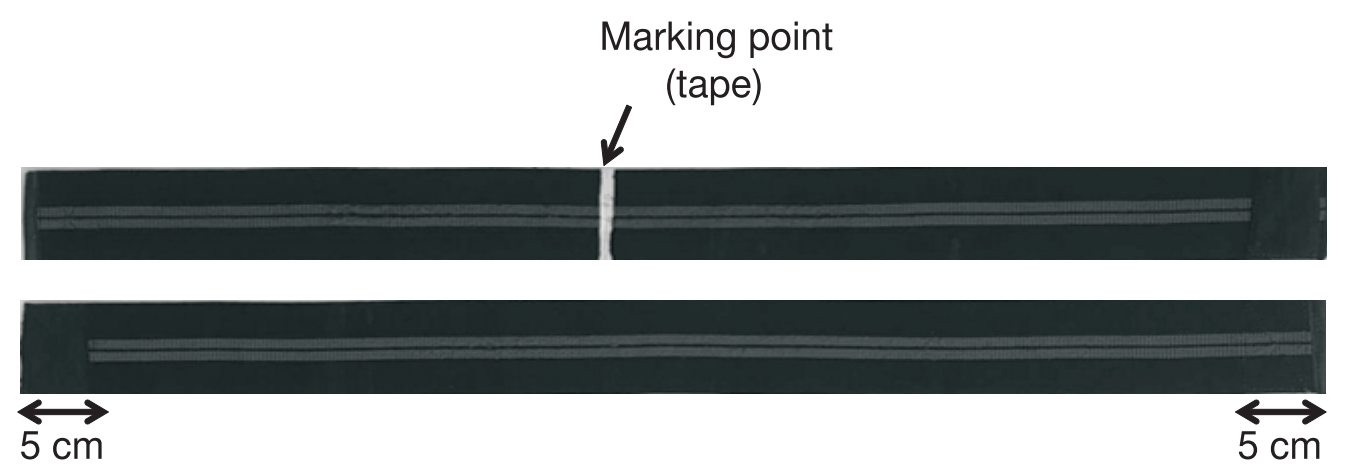

(Velcro tape)

(Velcro tape)

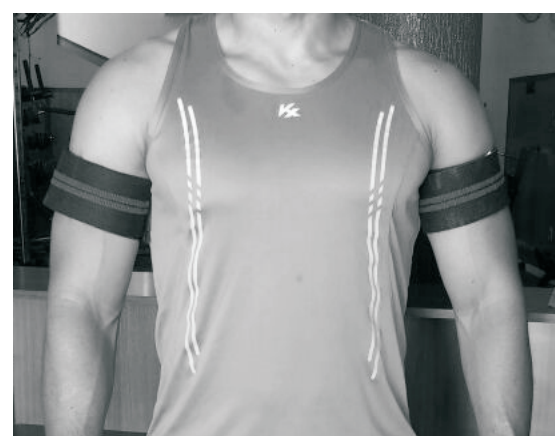

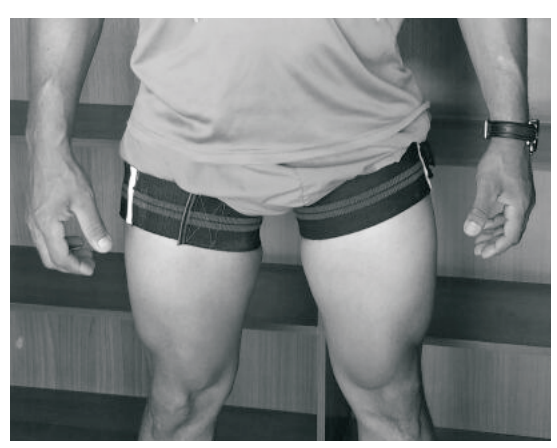

Figure 1. Blood flow restriction using $7.6 \mathrm{~cm}$ wide elastic knee wraps (Harbinger Red-Line, Fairfield, USA) adapted with Velcro tape $(5 \mathrm{~cm})$ at the extremities 
the subjects were queried regarding the perceived pressure. The reliability coefficients (ICC, intraclass correlation coefficient) for the perceived pressures were $0.74(p=0.014)$ for the arm and $0.86(p=0.001)$ for the thigh between the orientation and experimental sessions.

\section{Experimental sessions}

The participants arrived at the laboratory in a 3-4hour post-absorptive state, remained seated at rest for 10-15 min, and underwent scaling instructions and memory-anchoring procedures. Subsequently, RPE-AM, $\mathrm{RPE}-\mathrm{O}$, and [La] were measured, and the exercise protocol was initiated. [La] and RPE were measured during the exercises at the end of each set. A differentiated rating for RPE-AM (e.g., quadriceps/biceps) and an undifferentiated rating for RPE-O were determined. RPE-AM was always measured before RPE-O, as previously described [12, 14].

The experimental sessions (RE + BFR and TRE) consisted of arm curl and leg extension exercises. The participants performed 3 sets of 16 repetitions at 35\% of $1 \mathrm{RM}$ in RE + BFR and 3 sets of 8 repetitions at 70\% of 1RM in TRE. In both sessions, the exercises were performed with a repetition rate of 1 second for each concentric and eccentric phase with a 1-minute rest interval between each set and a 5-minute rest interval between exercises. The subjects were instructed to exhale during the concentric phase and inhale during the eccentric phase. Possible extraneous influences on perceptual responses were eliminated by blinding the participants regarding the weight lifted. In addition, the individuals were previously instructed to have the same food intake one day before and on the day of each experimental session.

\section{Statistical analysis}

Data are presented as means \pm standard deviations. The statistical significance of the results for all analyses was accepted at $p<0.05$. The perception variables (RPE-AM and RPE-O) were compared by using 2-way ANOVA (conditions [RE + BFR and TRE] $\times$ times [rest, set $1-\mathrm{S} 1$, set 2 - S2, and set 3 - S3]) with the Newman-Keuls post-hoc test. ANOVA was applied separately for each exercise type (arm curl and leg extension). The concurrent validation was determined via a linear regression analysis between [La] and the perception variables (RPE-AM and RPE-O). In this analysis, [La] was considered the independent variable, whereas RPE-AM and RPE-O were considered dependent variables. Separate linear regression analyses were calculated for each experimental condition (RE + BFR and TRE) and each exercise type (arm curl and leg extension). To meet the concurrent validation, a Pearson correlation coefficient $\geq 0.50$ (large effect size) was established as the cut-off point $[35,36]$.

\section{Ethical approval}

The research related to human use has complied with all the relevant national regulations and institutional policies, has followed the tenets of the Declaration of Helsinki, and has been approved by the Research Ethics Committee of the Integrated Colleges of Patos (CAAE: 27781014.6.0000.3181).

\section{Informed consent}

Informed consent has been obtained from all individuals included in this study.

\section{Results}

\section{Perceptual responses and [La]}

Table 1 shows the circumference of the upper and lower limbs and the elastic knee wrap used for BRF. The resistance loads differed between the conditions, both in arm curl (RE + BFR: $14.71 \pm 2.43$ vs. TRE: $29.56 \pm 4.76 \mathrm{~kg} ; p<0.01)$ and leg extension $(\mathrm{RE}+\mathrm{BFR}$ : $39.28 \pm 7.22$ vs. TRE: $77.87 \pm 14.58 \mathrm{~kg} ; p<0.01$ ). Table 2 presents the descriptive data of [La] at rest and the end of each set in RE + BFR and TRE for the 2 exercise types. [La] data were used inferentially in the

Table 1. Circumference of the upper and lower limbs and the elastic knee wrap used for blood flow restriction $(n=12)$

\begin{tabular}{lc}
\hline Variables & $\begin{array}{c}\text { Mean } \pm \text { standard } \\
\text { deviation }\end{array}$ \\
\hline Right thigh circumference $(\mathrm{cm})$ & $56.97 \pm 3.94$ \\
Left thigh circumference $(\mathrm{cm})$ & $56.47 \pm 4.34$ \\
Right arm circumference $(\mathrm{cm})$ & $33.56 \pm 3.53$ \\
Left arm circumference $(\mathrm{cm})$ & $33.43 \pm 3.30$ \\
ECBFRRT $(\mathrm{cm})$ & $39.84 \pm 2.72$ \\
ECBFRLT $(\mathrm{cm})$ & $39.29 \pm 2.50$ \\
ECBFRRA $(\mathrm{cm})$ & $25.17 \pm 2.64$ \\
ECBFRLA $(\mathrm{cm})$ & $24.98 \pm 2.70$ \\
\hline
\end{tabular}

ECBFRRT - elastic wrap circumference for blood flow restriction in the right thigh, ECBFRLT - elastic wrap circumference for blood flow restriction in the left thigh, ECBFRRA - elastic wrap circumference for blood flow restriction in the right arm, ECBFRLA - elastic wrap circumference for blood flow restriction in the left arm 
Table 2. Blood lactate concentration $\left(\mathrm{mmol} \cdot \mathrm{l}^{-1}\right)$ at rest and the end of each set in the RE + BFR and TRE exercises in the upper and lower limbs $(n=12)$

\begin{tabular}{|c|c|c|c|c|c|c|c|c|c|}
\hline \multirow{3}{*}{ Conditions } & \multirow{3}{*}{$n$} & \multirow{3}{*}{ Parameter } & \multicolumn{7}{|c|}{ Exercises/times } \\
\hline & & & \multirow{2}{*}{ Rest } & \multicolumn{3}{|c|}{ Arm curl } & \multicolumn{3}{|c|}{ Leg extension } \\
\hline & & & & $\mathrm{S} 1$ & $\mathrm{~S} 2$ & S3 & S1 & $\mathrm{S} 2$ & S3 \\
\hline \multirow{2}{*}{$\mathrm{RE}+\mathrm{BFR}$} & \multirow{2}{*}{12} & Mean & 1.82 & 4.59 & 5.10 & 6.46 & 4.13 & 5.11 & 6.73 \\
\hline & & $S D$ & 0.60 & 2.85 & 2.56 & 2.58 & 2.58 & 1.94 & 1.95 \\
\hline \multirow{2}{*}{ TRE } & \multirow{2}{*}{12} & Mean & 1.46 & 3.45 & 4.60 & 5.65 & 4.20 & 5.58 & 6.67 \\
\hline & & $S D$ & 0.51 & 1.91 & 1.56 & 1.24 & 2.96 & 3.20 & 2.80 \\
\hline
\end{tabular}

$\mathrm{RE}+\mathrm{BFR}$ - resistance exercise with blood flow restriction, TRE - traditional resistance exercise, $\mathrm{S}$ - set,

$S D$ - standard deviation

Table 3. Rating of perceived exertion in the active muscles (OMNI-RES) at rest and the end of each set in the RE + BFR and TRE exercises in the upper and lower limbs $(n=12)$

\begin{tabular}{|c|c|c|c|c|c|c|c|c|c|}
\hline \multirow{3}{*}{ Conditions } & \multirow{3}{*}{$n$} & \multirow{3}{*}{ Parameter } & \multicolumn{7}{|c|}{ Exercises/times } \\
\hline & & & \multirow{2}{*}{ Rest } & \multicolumn{3}{|c|}{ Arm curl } & \multicolumn{3}{|c|}{ Leg extension } \\
\hline & & & & S1 & S2 & S3 & S1 & S2 & S3 \\
\hline \multirow{2}{*}{$\mathrm{RE}+\mathrm{BFR}$} & \multirow{2}{*}{12} & Mean & 0.16 & $6.33 * \$$ & $8.25^{*} \dagger$ & $9.41 *+\$$ & $6.25 * \$$ & $7.91 * \dagger$ & $9.16^{*}+\$$ \\
\hline & & $S D$ & 0.38 & 1.77 & 0.62 & 0.66 & 1.81 & 1.24 & 0.71 \\
\hline \multirow{2}{*}{ TRE } & \multirow[b]{2}{*}{12} & Mean & 0.08 & $7.33^{*}$ & $8.08^{*} \dagger$ & $8.66^{*}$ & $7.00^{*}$ & $7.58^{*}$ & $8.25^{*}$ \\
\hline & & $S D$ & 0.28 & 1.43 & 0.99 & 1.07 & 1.53 & 1.78 & 1.60 \\
\hline
\end{tabular}

$\mathrm{RE}+\mathrm{BFR}$ - resistance exercise with blood flow restriction, TRE - traditional resistance exercise, $\mathrm{S}$ - set,

$S D$ - standard deviation

* significant difference $(p<0.05)$ compared with the rest, $\dagger$ significant difference $(p<0.05)$ compared with the previous set, $\$$ significant difference $(p<0.05)$ compared with TRE

Table 4. Rating of perceived exertion in the overall body (OMNI-RES) at rest and the end of each set in the RE + BFR and TRE exercises in the upper and lower limbs $(n=12)$

\begin{tabular}{|c|c|c|c|c|c|c|c|c|c|}
\hline \multirow{3}{*}{ Conditions } & \multirow{3}{*}{$n$} & \multirow{3}{*}{ Parameter } & \multicolumn{7}{|c|}{ Exercises/times } \\
\hline & & & \multirow{2}{*}{ Rest } & \multicolumn{3}{|c|}{ Arm curl } & \multicolumn{3}{|c|}{ Leg extension } \\
\hline & & & & $\mathrm{S} 1$ & $\mathrm{~S} 2$ & S3 & $\mathrm{S} 1$ & $\mathrm{~S} 2$ & S3 \\
\hline \multirow{2}{*}{$\mathrm{RE}+\mathrm{BFR}$} & \multirow{2}{*}{12} & Mean & 0.16 & $6.25^{*} \$$ & $8.00 * \dagger$ & $8.66^{*}$ & $5.50 *$ & $7.25^{*} \dagger$ & $8.33^{*} \dagger$ \\
\hline & & $S D$ & 0.38 & 1.60 & 0.85 & 0.98 & 2.35 & 1.54 & 1.23 \\
\hline \multirow{2}{*}{ TRE } & \multirow{2}{*}{12} & Mean & 0.16 & $7.33^{*}$ & $7.66^{*}$ & $8.41^{*} \dagger$ & $6.41^{*}$ & $7.16^{*} \dagger$ & $7.91 * \dagger$ \\
\hline & & $S D$ & 0.38 & 1.37 & 1.15 & 1.24 & 1.72 & 1.69 & 1.88 \\
\hline
\end{tabular}

$\mathrm{RE}+\mathrm{BFR}$ - resistance exercise with blood flow restriction, TRE - traditional resistance exercise, $\mathrm{S}$ - set,

$S D$ - standard deviation

* significant difference $(p<0.05)$ compared with the rest, $\dagger$ significant difference $(p<0.05)$ compared with the previous set,

$\$$ significant difference $(p<0.05)$ compared with TRE

regression analysis to examine the concurrent validity of the OMNI-RES because [La] was a criterion variable (see Table 5).

Results indicated that RPE-AM (Table 3) and RPE-O (Table 4) were not significantly different between RE + BFR and TRE at rest in the 2 exercise types. In the comparison between RE + BFR and TRE, RPE-AM was higher during S1 in TRE. However, RPE-AM was higher during S3 in RE + BFR than TRE in both exercise types $(p<0.05)$. Throughout the sessions, RPE$\mathrm{AM}$ increased in RE + BFR from S1 to S2 and from S2 to S3 $(p<0.05)$ in both exercise types (Table 3).

With regard to RPE-O, there was a significant difference between RE + BFR and TRE only at $\mathrm{S} 1$ for the arm curl, and the values were higher for TRE $(p<0.05)$. In the exercise sessions, RPE-O increased from S1 to 
Table 5. Linear regression analysis of RPE expressed as a function of [La] during RE + BFR and TRE exercises in the upper and lower limbs $(n=12)$

\begin{tabular}{|c|c|c|c|c|c|c|c|c|c|c|}
\hline \multirow[b]{2}{*}{ Conditions } & \multirow[b]{2}{*}{$n$} & \multirow[b]{2}{*}{ Exercises } & \multicolumn{2}{|c|}{ Variable } & \multirow[b]{2}{*}{ Intercept } & \multirow[b]{2}{*}{$S E$} & \multirow[b]{2}{*}{ Slope } & \multirow[b]{2}{*}{$S E$} & \multirow[b]{2}{*}{$r^{*}$} & \multirow[b]{2}{*}{$r^{2}$} \\
\hline & & & $\begin{array}{c}\text { Criterion } \\
\mathrm{X}\end{array}$ & $\begin{array}{c}\text { Concurrent } \\
\text { Y }\end{array}$ & & & & & & \\
\hline \multirow{2}{*}{$\mathrm{RE}+\mathrm{BFR}$} & \multirow{2}{*}{12} & \multirow{2}{*}{$\mathrm{AC}$} & \multirow{2}{*}{ [La] } & RPE-AM & 2.774 & 0.921 & 0.728 & 0.174 & 0.54 & 0.29 \\
\hline & & & & RPE-O & 2.647 & 0.871 & 0.695 & 0.165 & 0.55 & 0.30 \\
\hline \multirow{2}{*}{ TRE } & \multirow{2}{*}{12} & \multirow{2}{*}{$\mathrm{AC}$} & \multirow{2}{*}{ [La] } & RPE-AM & 1.781 & 0.924 & 1.118 & 0.214 & 0.63 & 0.39 \\
\hline & & & & RPE-O & 1.942 & 0.916 & 1.033 & 0.212 & 0.60 & 0.36 \\
\hline \multirow{2}{*}{$\mathrm{RE}+\mathrm{BFR}$} & \multirow{2}{*}{12} & \multirow{2}{*}{ LE } & \multirow{2}{*}{ [La] } & RPE-AM & 1.276 & 0.811 & 1.023 & 0.158 & 0.71 & 0.50 \\
\hline & & & & RPE-O & 0.736 & 0.740 & 1.003 & 0.144 & 0.74 & 0.54 \\
\hline \multirow{2}{*}{ TRE } & \multirow{2}{*}{12} & \multirow{2}{*}{ LE } & \multirow{2}{*}{ [La] } & RPE-AM & 2.528 & 0.755 & 0.724 & 0.138 & 0.63 & 0.40 \\
\hline & & & & RPE-O & 2.625 & 0.753 & 0.657 & 0.137 & 0.59 & 0.35 \\
\hline
\end{tabular}

RPE - rating of perceived exertion (OMNI-RES) in the active muscles (RPE-AM) and in the overall body (RPE-O), $\mathrm{RE}+\mathrm{BFR}$ - resistance exercise with blood flow restriction, TRE - traditional resistance exercise, $S E$ - standard error, AC - arm curl, LE - leg extension, [La] - blood lactate concentration $\left(\mathrm{mmol} \cdot \mathrm{l}^{-1}\right)$ ${ }^{*} p<0.01$

$\mathrm{S} 2$ and from S2 to S3 for leg extension in both RE + BFR and TRE $(p<0.05)$ (Table 4$)$.

\section{Concurrent validation}

In the RE $+\mathrm{BFR}$, positive regression coefficients were found $(p<0.01)$ between [La] and RPE-AM (arm curl: $r=0.54$; leg extension: $r=0.71$ ) and between [La] and RPE-O (arm curl: $r=0.55$; leg extension: $r=0.74)$. Similarly, for TRE, positive regression coefficients $(p<0.01)$ were found between $[\mathrm{La}]$ and RPE-AM (arm curl: $r=0.63$; leg extension: $r=0.63$ ) and between [La] and RPE-O (arm curl: $r=0.60$; leg extension: $r=0.59)$ (Table 5).

\section{Discussion}

This study examined the concurrent validity of RPE for RE + BFR in the upper and lower limbs. Our main findings were that both RPE-AM and RPE-O increased as a function of time during RE + BFR sessions in both the arm curl and leg extension exercise. Furthermore, a positive correlation was found between [La] and RPE-AM and between [La] and RPE-O in both exercise types in RE + BFR. The findings are consistent with the predictions of Borg's Effort Continua Model [37], which establishes a functional correlation between the 3 most critical responses to effort - physiological, perceptual, and performance; it is proposed that RPE is a valid measure to monitor and regulate the intensity of the load undertaken during $\mathrm{RE}+\mathrm{BFR}$ sessions.
The correlation between RPE and other variables (e.g., [La], load) establishes that perceived effort is valid to evaluate the intensity of RE + BFR and TRE sessions. This study was the first to correlate RPE with [La] during RE + BFR sessions, finding a significant positive correlation between lactacidosis and both RPE-AM $(r=0.54 ; r=0.71)$ and RPE-O $(r=0.55 ; r=$ $0.74)$ for arm curl and leg extension exercises, respectively. These results are corroborated by previous studies that used RE without BFR, including that by Aniceto et al. [11], wherein a positive correlation was observed between RPE-AM and [La] during a TRE session and circuit weight training ( $r=0.65$ and $r=0.56$, respectively). Robertson et al. [12] reported a strong correlation $(r=0.87)$ between [La] and RPE measured immediately after arm curls. Similarly, Kraemer et al. [38] found a positive correlation $(r=0.84)$ between [La] and RPE during a session of 10 REs with 3 sets of 10 maximum repetitions. By contrast, other studies did not find a significant correlation $(p>0.05)$ between $[\mathrm{La}]$ and RPE after a single set of arm curls at different loads [15] or during and after RE sessions with different orders [39].

Correlation coefficients are an appropriate method to assess the concurrent validity of perceived exertion [12, 14]. However, these coefficients decrease in cases of limited inter-individual variance [40, 41]. One of the factors that may have limited the variance among participants was the type of experimental design used. This study applied a within-subject repeated measures crossover design involving a single group of individuals who were subjected to the same experi- 
mental conditions throughout all sessions. Another aspect that may have influenced the correlation coefficients was that many subjects responded with the same or a similar exertional rating at a given time. It should be noted that RPE (OMNI-RES) is a categorical variable, and this may have resulted in limited variation among the participants [30], in contrast to [La], for which practically no results were equal among the participants. However, it is important to mention that the correlations found in RE + BFR and TRE represent a large effect size $(r \geq 0.50)$ in accordance with Cohen [35] and Hopkins et al. [36] and a very large effect size $(r \geq 0.70)$ in accordance with Hopkins et al. [36]. Correlation coefficients higher than 0.70 between RPE and [La] were found only in the leg extension exercise during the RE + BFR sessions. This result may be caused by the fact that the thigh segment has a greater muscle mass and the sitting position on the leg extension machine enhances BFR in the lower limbs, leading to a greater change in [La] and RPE.

In the arm curl and leg extension exercises, our results indicated that at the end of S1, RPE-AM was higher in TRE compared with RE + BFR. However, at the end of S2, no significant difference was found between these 2 exercise conditions, and after S3, RPE-AM values in RE + BFR were higher than in TRE. This change of RPE throughout the exercise session was differentiated between TRE and RE + BFR, possibly because TRE promotes a predominantly mechanical stress (strain), whereas RE + BFR primarily induces a metabolic stress [42].

RPE-AM appears to be the main determinant of perceived effort during RE + BFR and TRE, with a predominance of local muscle signals on the sensory process. Studies have shown that localized muscle tension is a strong indicator of perceived exertion. Therefore, muscle (muscle spindles) and tendon (Golgi tendon organs) sensors are primarily responsible for the perceived effort together with the metabolic cost of the mechanical work performed $[15,43]$. This may explain the data from the present study, wherein after S1 in the arm curl and leg extension exercises, RPE-AM was higher in TRE than in RE + BFR. However, at the end of S3, RPE-AM was higher in RE + BFR and this response may be a result of the use of the elastic wraps, which caused hypoxia because of the decreased blood flow in the exercised muscles and led to the increased accumulation of [La] together with other metabolites, such as $\mathrm{H}^{+}$ions $[1,2,22,23,33]$ and inorganic phosphate $[42,44]$. These metabolic changes, along with the discomfort caused by the application of elastic wraps to the limbs [4], may have induced a cu- mulative fatigue effect during the RE + BFR session and consecutively increased RPE-AM and RPE-O. Because TRE does not use BFR, the resynthesis of adenosine triphosphate-creatine phosphate (ATP-CP) occurs partially during the recovery interval between $\mathrm{RE}$ sets ( 1 minute) and decreases the cumulative effect of fatigue throughout the session.

As noted above, the results of the present study validate the use of RPE-AM and RPE-O (OMNI-RES) to monitor the intensity of RE + BFR and TRE with upper and lower body protocols. In addition, the present investigation brings new information on the prescription of BRF using elastic knee wraps with regard to the pressure exerted by the wrap and on its application to the upper and lower limbs, which favours the use of RE + BFR in various settings (e.g., sports centres, gyms, clinics). In addition, BFR with elastic knee wraps has been used in previous studies $[4,5,19$, 45-47]. However, those studies have shown a limitation to this technique regarding the BFR pressure exerted by the elastic wrap, considering that the researchers applied the wrap without using a reference criterion and only reported placing the elastic wrap by the same investigator to maximize intra-rater reliability.

\section{Conclusions}

Our results indicate that RPE in the active muscles and the overall body is a valid method to monitor the intensity of effort during RE + BFR of the upper and lower limbs because it was positively correlated with [La] during the exercise sessions. Therefore, RPE can be used to prescribe and control the intensity of effort in RE + BFR sessions without the need for invasive methods (e.g., [La]). However, further studies should be conducted in other populations (e.g., older adults) to assess whether RPE is sensitive to different BFR pressures and/or wrap/cuff widths.

\section{Acknowledgements}

The authors are grateful to the professors Dr. Wagner Prado and Dr. Mauro Barros of the Associate Graduate Program in Physical Education UPE/UFPB for providing the YSI 1500 Sport Lactate Analyzer, to the Coordination for the Improvement of Higher Education Personnel (CAPES, Brazil) for the doctor's scholarship, and to all of the participants for their efforts and commitment during the study period.

\section{Disclosure statement}

No author has any financial interest or received any financial benefit from this research. 


\section{Conflict of interest}

The authors state no conflict of interest.

\section{References}

1. Loenneke JP, Wilson GJ, Wilson JM. A mechanistic approach to blood flow occlusion. Int J Sports Med. 2010;31(1):1-4; doi: 10.1055/s-0029-1239499.

2. Pope ZK, Willardson JM, Schoenfeld BJ. Exercise and blood flow restriction. J Strength Cond Res. 2013;27(10): 2914-2926; doi: 10.1519/JSC.0b013e3182874721.

3. Hollander DB, Reeves GV, Clavier JD, Francois MR, Thomas C, Kraemer RR. Partial occlusion during resistance exercise alters effort sense and pain. J Strength Cond Res. 2010;24(1):235-243; doi: 10.1519/JSC. 0b013e3181c7badf.

4. Loenneke JP, Balapur A, Thrower AD, Barnes JT, Pujol TJ. The perceptual responses to occluded exercise. Int J Sports Med. 2011;32(3):181-184; doi: 10.1055/s0030-1268472.

5. Loenneke JP, Kearney ML, Thrower AD, Collins S, Pujol TJ. The acute response of practical occlusion in the knee extensors. J Strength Cond Res. 2010;24(10): 2831-2834; doi: 10.1519/JSC.0b013e3181f0ac3a.

6. Loenneke JP, Pujol TJ. The use of occlusion training to produce muscle hypertrophy. Strength Cond J. 2009; 31(3):77-84; doi: 10.1519/SSC.0b013e3181a5a352.

7. Loenneke JP, Wilson JM, Marín PJ, Zourdos MC, Bemben MG. Low intensity blood flow restriction training: a meta-analysis. Eur J Appl Physiol. 2012;112(5): 1849-1859; doi: 10.1007/s00421-011-2167-x.

8. Yasuda T, Loenneke JP, Thiebaud RS, Abe T. Effects of blood flow restricted low-intensity concentric or eccentric training on muscle size and strength. PLoS One. 2012;7(12):e52843; doi: 10.1371/journal.pone.0052843.

9. Yasuda T, Ogasawara R, Sakamaki M, Ozaki H, Sato Y, Abe T. Combined effects of low-intensity blood flow restriction training and high-intensity resistance training on muscle strength and size. Eur J Appl Physiol. 2011;111(10):2525-2533; doi: 10.1007/s00421-0111873-8.

10. Ewing Garber C, Blissmer B, Deschenes MR, Franklin BA, Lamonte MJ, Lee I-M, et al. American College of Sports Medicine position stand. Quantity and quality of exercise for developing and maintaining cardiorespiratory, musculoskeletal, and neuromotor fitness in apparently healthy adults: guidance for prescribing exercise. Med Sci Sports Exerc. 2011;43(7):1334-1359; doi: 10.1249/MSS.0b013e318213fefb.

11. Aniceto RR, Ritti-Dias RM, Dos Prazeres TM, Farah BQ, de Lima FFM, do Prado WL. Rating of perceived exertion during circuit weight training: a concurrent validation study. J Strength Cond Res. 2015;29(12):33363342; doi: 10.1519/jsc.0000000000000998.

12. Robertson RJ, Goss FL, Rutkowski J, Lenz B, Dixon C, Timmer J, et al. Concurrent validation of the OMNI per- ceived exertion scale for resistance exercise. Med Sci Sports Exerc. 2003;35(2):333-341; doi: 10.1249/01. mss.0000048831.15016.2a.

13. Suminski RR, Robertson RJ, Arslanian S, Kang J, Utter AC, DaSilva SG, et al. Perception of effort during resistance exercise. J Strength Cond Res. 1997;11(4): 261-265.

14. Lagally KM, Robertson RJ. Construct validity of the OMNI resistance exercise scale. J Strength Cond Res. 2006;20(2):252-256; doi: 10.1519/r-17224.1.

15. Lagally KM, Robertson RJ, Gallagher KI, Goss FL, Jakicic JM, Lephart SM, et al. Perceived exertion, electromyography, and blood lactate during acute bouts of resistance exercise. Med Sci Sports Exerc. 2002;34(3): 552-559; doi: 10.1097/00005768-200203000-00025.

16. Gearhart Jr RF, Goss FL, Lagally KM, Jakicic JM, Gallagher J, Gallagher KI, et al. Ratings of perceived exertion in active muscle during high-intensity and low-intensity resistance exercise. J Strength Cond Res. 2002;16(1):87-91; doi: 10.1519/00124278-20020200000013.

17. Eston R, Evans HJL. The validity of submaximal ratings of perceived exertion to predict one repetition maximum. J Sports Sci Med. 2009;8(4):567-573.

18. Robertson RJ, Goss FL, Aaron DJ, Nagle EF, Gallagher M Jr., Kane IR, et al. Concurrent muscle hurt and perceived exertion of children during resistance exercise. Med Sci Sports Exerc. 2009;41(5):1146-1154; doi: 10.1249/MSS.0b013e3181930321.

19. Loenneke JP, Balapur A, Thrower AD, Barnes J, Pujol TJ. Blood flow restriction reduces time to muscular failure. Eur J Sport Sci. 2012;12(3):238-243; doi: 10.1080/ 17461391.2010.551420.

20. Pinto RR, Karabulut M, Poton R, Polito MD. Acute resistance exercise with blood flow restriction in elderly hypertensive women: haemodynamic, rating of perceived exertion and blood lactate. Clin Physiol Funct Imaging. 2018;38(1):17-24; doi: 10.1111/cpf.12376.

21. Wernbom M, Järrebring R, Andreasson MA, Augustsson J. Acute effects of blood flow restriction on muscle activity and endurance during fatiguing dynamic knee extensions at low load. J Strength Cond Res. 2009; 23(8):2389-2395; doi: 10.1519/JSC.0b013e3181bc1c2a.

22. Yasuda T, Abe T, Brechue WF, Iida H, Takano H, Meguro $\mathrm{K}$, et al. Venous blood gas and metabolite response to low-intensity muscle contractions with external limb compression. Metabolism. 2010;59(10):15101519; doi: 10.1016/j.metabol.2010.01.016.

23. Yasuda T, Fukumura K, Iida H, Nakajima T. Effect of low-load resistance exercise with and without blood flow restriction to volitional fatigue on muscle swelling. Eur J Appl Physiol. 2015;115(5):919-926; doi: 10.1007/s00421-014-3073-9.

24. Beck TW. The importance of a priori sample size estimation in strength and conditioning research. J Strength Cond Res. 2013;27(8):2323-2337; doi: 10.1519/JSC. ob013e318278eea0. 
25. Faul F, Erdfelder E, Buchner A, Lang A-G. Statistical power analyses using $G^{*}$ Power 3.1: tests for correlation and regression analyses. Behav Res Methods. 2009; 41(4):1149-1160; doi: 10.3758/brm.41.4.1149.

26. Aniceto RR, Ritti-Dias RM, Scott CB, Matrins de Lima FF, dos Prazeres TMP, do Prado WL. Acute effects of different weight training methods on energy expenditure in trained men. Rev Bras Med Esporte. 2013;19(3):181-185.

27. Jackson AS, Pollock ML. Generalized equations for predicting body density of men. Br J Nutr. 1978;40(3): 497-504; doi: 10.1079/bjn19780152.

28. Kraemer WJ, Ratamess NA, Fry AC, French DN. Strength testing: development and evaluation of methodology. In: Maud PJ, Foster C (eds.), Physiological assessment of human fitness. Champaign: Human Kinetics; 2006; 119-150.

29. Gearhart RE, Goss FL, Lagally KM, Jakicic JM, Gallagher J, Robertson RJ. Standardized scaling procedures for rating perceived exertion during resistance exercise. J Strength Cond Res. 2001;15(3):320-325; doi: 10.1519/1533-4287(2001)015<0320:SSPFRP>2.0.CO;2.

30. Lagally KM, Costigan EM. Anchoring procedures in reliability of ratings of perceived exertion during resistance exercise. Percept Mot Skills. 2004;98(3 Pt 2): 1285-1295; doi: 10.2466/pms.98.3c.1285-1295.

31. Jessee MB, Buckner SL, Dankel SJ, Counts BR, Abe T, Loenneke JP. The influence of cuff width, sex, and race on arterial occlusion: implications for blood flow restriction research. Sports Med. 2016;46(6):913-921; doi: 10.1007/s40279-016-0473-5.

32. Loenneke JP, Allen KM, Mouser JG, Thiebaud RS, Kim D, Abe T, et al. Blood flow restriction in the upper and lower limbs is predicted by limb circumference and systolic blood pressure. Eur J Appl Physiol. 2015; 115(2):397-405; doi: 10.1007/s00421-014-3030-7.

33. Loenneke JP, Fahs CA, Rossow LM, Sherk VD, Thiebaud RS, Abe T, et al. Effects of cuff width on arterial occlusion: implications for blood flow restricted exercise. Eur J Appl Physiol. 2012;112(8):2903-2912; doi: 10.1007/s00421-011-2266-8.

34. Wilson JM, Lowery RP, Joy JM, Loenneke JP, Naimo MA. Practical blood flow restriction training increases acute determinants of hypertrophy without increasing indices of muscle damage. J Strength Cond Res. 2013;27(11):3068-3075; doi: 10.1519/JSC.0b013 e31828a1ffa.

35. Cohen J. A power primer. Psychol Bull. 1992;112(1): 155-159; doi: 10.1037//0033-2909.112.1.155.

36. Hopkins WG, Marshall SW, Batterham AM, Hanin J. Progressive statistics for studies in sports medicine and exercise science. Med Sci Sports Exerc. 2009;41(1):313; doi: 10.1249/MSS.0b013e31818cb278.

37. Borg G. Perceived exertion as an indicator of somatic stress. Scand J Rehabil Med. 1970;2(2):92-98; doi: 10.2340/1650197719702239298.
38. Kraemer WJ, Noble BJ, Clark MJ, Culver BW. Physiologic responses to heavy-resistance exercise with very short rest periods. Int J Sports Med. 1987;8(4):247-252; doi: 10.1055/s-2008-1025663.

39. Bellezza PA, Hall EE, Miller PC, Bixby WR. The influence of exercise order on blood lactate, perceptual, and affective responses. J Strength Cond Res. 2009; 23(1):203-208; doi: 10.1519/jsc.0b013e3181889156.

40. Denegar CR, Ball DW. Assessing reliability and precision of measurement: an introduction to intraclass correlation and standard error of measurement. J Sport Rehabil. 1993;2(1):35-42; doi: 10.1123/jsr.2.1.35.

41. Lamb KL. Children's ratings of effort during cycle ergometry: an examination of the validity of two effort rating scales. Pediatr Exerc Sci. 1995;7(4):407-421; doi: 10.1123/pes.7.4.407.

42. Takada S, Okita K, Suga T, Omokawa M, Kadoguchi T, Sato T, et al. Low-intensity exercise can increase muscle mass and strength proportionally to enhanced metabolic stress under ischemic conditions. J Appl Physiol. 2012;113(2):199-205; doi: 10.1152/japplphysiol.00149. 2012.

43. Mihevic PM. Sensory cues for perceived exertion: a review. Med Sci Sports Exerc. 1981;13(3):150-163.

44. Harris RC, Edwards RH, Hultman E, Nordesjö LO, Nylind B, Sahlin K. The time course of phosphorylcreatine resynthesis during recovery of the quadriceps muscle in man. Pflugers Arch. 1976;367(2):137-142; doi: 10.1007/BF00585149.

45. Loenneke JP, Thrower AD, Balapur A, Barnes JT, Pujol TJ. Blood flow-restricted walking does not result in an accumulation of metabolites. Clin Physiol Funct Imaging. 2012;32(1):80-82; doi: 10.1111/j.1475-097X. 2011.01059.x.

46. Loenneke JP, Wilson JM, Balapur A, Thrower AD, Barnes JT, Pujol TJ. Time under tension decreased with blood flow-restricted exercise. Clin Physiol Funct Imaging. 2012;32(4):268-273; doi: 10.1111/j.1475-097X. 2012.01121.x.

47. Loenneke JP, Young KC, Wilson JM, Andersen JC. Rehabilitation of an osteochondral fracture using blood flow restricted exercise: a case review. J Bodyw Mov Ther. 2013;17(1):42-45; doi: 10.1016/j.jbmt.2012.04. 006. 